Short communication

\title{
Clustering of HTLV-1 associated myelopathy/tropical spastic paraparesis (HAM/TSP) and infective dermatitis associated with HTLV-1 (IDH) in Salvador, Bahia, Brazil
}

\author{
José Lucas Sena da Silva ${ }^{a, c}$, Janeusa Rita L. Primo ${ }^{\mathrm{b}}$, Maria de Fátima S.P. de Oliveira ${ }^{\mathrm{b}}$, \\ Everton da Silva Batista ${ }^{a}$, Otávio Moreno-Carvalho ${ }^{\mathrm{b}}$, Lourdes Farrée ${ }^{\mathrm{c}, \mathrm{d}}$, \\ Achiléa L. Bittencourta,* \\ a Department of Pathology, Professor Edgard Santos Teaching Hospital, Federal University of Bahia, Salvador, Bahia, Brazil \\ ${ }^{\mathrm{b}}$ Department of Internal Medicine, Professor Edgard Santos Teaching Hospital, Federal University of Bahia, Salvador, Bahia, Brazil \\ ${ }^{\mathrm{c}}$ Laboratory of Experimental Pathology, Gonçalo Muniz Researcher Center, Oswaldo Cruz Foundation, Salvador, Bahia, Brazil \\ ${ }^{\mathrm{d}}$ National Institute of Science and Technology of Tropical Diseases, Brazil
}

\section{A R T I C L E I N F O}

\section{Article history:}

Received 15 March 2013

Received in revised form 12 July 2013

Accepted 16 July 2013

\section{Keywords:}

HTLV-1 infection

Clustering of HTLV-1-associated diseases

HTLV-1 associated myelopathy/tropical

spastic paraparesis

Infective dermatitis associated with HTLV-1

HTLV-1 vertical transmission

\begin{abstract}
A B S T R A C T
Fifteen families with clustering of infective dermatitis associated with HTLV-1 (IDH) and/or HTLV-1 associated myelopathy/tropical spastic paraparesis (HAM/TSP) were observed among 28 families of IDH index cases, $93 \%$ of them occurring in two generations. With the exception of two mothers of children with IDH, all the mothers with HAM/TSP had at least one child with HAM/TSP. This is the first report of such clustering involving many families.
\end{abstract}

(C) 2013 Elsevier B.V. All rights reserved.

\section{Introduction}

The human T-cell lymphotropic virus type 1 (HTLV-1) may cause, among other diseases, HTLV-1-associated myelopathy/ tropical spastic paraparesis (HAM/TSP), and infective dermatitis associated to HTLV-1 (IDH) [1]. HAM/TSP was generally considered to be the result of HTLV-1 infection acquired in adult life [2]. Notwithstanding there are a few reports in the literature about the infantile and juvenile form of HAM/TSP in which the infection is acquired vertically [1]. Most of these cases were associated with IDH [3].

The development of HAM/TSP in HTLV-1 carriers is not frequent, occurring in about $1-2 \%$ of carriers. There are rare reports of familial clustering of the adult form of HAM/TSP in siblings [4], or in more than one generation [5,6]. In Colombia, McKhann et al. [7] described one family, the father, the mother and their 13-year-old

\footnotetext{
* Corresponding author at: Laboratório de Patologia, Complexo Hospitalar Universitário Prof. Edgard Santos, Universidade Federal da Bahia, Rua Dr. Augusto Viana, s/n, Canela, CEP: 40.110-060 Salvador, Bahia, Brazil. Tel.: +55 713283 8016; fax: +557132838016 .

E-mail address: achilea@uol.com.br (A.L. Bittencourt).
}

child with definite HAM/TSP. Clustering of ATL with HAM/TSP in adults has been described in Brazil, in Rio de Janeiro [6], Jamaica [8] and Martinique [9].

In the current study, families of 28 IDH pediatric patients were investigated and clustering of IDH and/or HAM/TSP was described in 15 of them.

\section{Materials and methods}

\subsection{Study population}

Families of 28 IDH patients were considered in this study. Besides the IDH cases, the 28 mothers, 90 siblings, and 32 fathers were also contacted. In four families the mother had more than one partner. In total, 60 siblings and seven fathers agreed to participate of this study. Vertical transmission of HTLV-1 for IDH patients and HTLV-1 positive serology for the mothers were considered as including criteria. The IDH patients were previously diagnosed during childhood and adolescence at the Dermatology Outpatient Clinic of the Federal University of Bahia, Brazil according to established criteria (Table $1 ;[3]$ ) and were considered as the index cases of the families. The presence of HTLV-1/2 antibodies in the index 
Table 1

Major criteria for diagnosis of infective dermatitis associated with human T-cell lymphotropic virus type $1(\mathrm{HTLV}-1)$ [3].

1. Presence of erythematous-scaly, exudative, and crusted lesions of the scalp, retroauricular areas, neck, axillae, groin, paranasal and perioral skin, ears, thorax, abdomen, and other sites.

2. Crusting of nostrils.

3. Chronic relapsing dermatitis with prompt response to appropriate therapy but prompt recurrence on discontinuation of antibiotics.

4. Diagnosis of HTLV-1 infection (by serological or molecular biological testing).

Of the 4 major criteria, 3 are required for diagnosis, with mandatory inclusion of 1,3 , and 4 . To fulfill criteria 1 , involvement of $\geq 3$ of the sites is required, including involvement of the scalp and retroauricular areas.

cases and their relatives was investigated by serology using the enzyme-linked immunosorbent assay (ELISA, Murex Biotech) and the diagnosis was confirmed by a Western blot, which is capable of discriminating between HTLV-1 and HTLV-2 (HTLV blot 2.4, Genelabs, Singapore) and/or a laboratory validated polymerase chain reaction (PCR) assay targeting HTLV-1 tax gene in peripheral blood mononuclear cells. This PCR assay was performed in all the index cases and in their siblings. All the HTLV-1 positive members of these families were screened by serology for HIV types 1 and 2 (ELISA, DiaSorin, Italy), underwent routine laboratory testing and clinical examination that included dermatological and neurological evaluations. All of them answered an epidemiological questionnaire. In the children and adolescents, obstetric and developmental milestones data were collected. The neurological diagnosis was based on clinical features and laboratory findings and was made in accordance with the WHO diagnostic guidelines for HAM/TSP [10]. This disease was classified as definite, probable or possible according to previously established criteria (Table 2) [11]. For those patients suspected to have HAM/TSP, the following laboratory tests were performed: urine culture, parasitological stool examination, complete blood cell count, and measurement of serum levels of IgA, IgE, IgG, IgM, calcium, phosphorus, albumin, glutamicoxalacetic/pyruvic transaminases, and alkaline phosphatase. Cell counts and total protein and immunoglobulin levels were measured on the cerebrospinal fluid (CSF). Analysis for antibodies against HTLV-1 and the infectious agents of toxoplasmosis, cysticercosis, syphilis, and schistosomiasis was also performed on the CSF. Additionally, the presence of HTLV-1 infected cells in the CSF was determined by PCR analysis using DNA amplification of proviral tax sequences as described above. In the current study, a family cluster was defined as the presence of more than one member with IDH and/or HAM/TSP. All the index cases and their siblings had been breast-fed. The study was approved by the Institutional Review Board of the Hospital Climério de Oliveira of the Federal University of Bahia, and informed consent was obtained in all cases.

\section{Results}

No case of infection by HTLV-2, HIV-1 and HIV-2 was observed. The index and sibling cases with HAM/TSP were followed up from 2 to 14 years (mean: 9.9 years). All the patients with HAM/TSP presented a non-remitting progressive spastic paraparesis with impaired gait. Urinary symptoms or signs were observed in the patients with longer evolution. Fifteen of the 28 IDH cases developed HAM/TSP, but 3 did not have CSF examinations. From the 60 siblings screened by serology for HTLV-1, 22 (36.7\%) were positive. Among the 22 infected siblings, 10 (10/22) developed IDH and/or HAM/TSP: 4 definite HAM/TSP, 3 possible HAM/TSP, 2 an association of IDH and definite HAM/TSP and 1 IDH. From the 28 seropositive mothers, 14 (50\%) had one of these diseases: 9 definite HAM/TSP, 4 possible HAM/TSP and 1 IDH and definite HAM/TSP (Fig. 1). All 7 fathers tested were HTLV-1 positive. Of these, 2 had possible HAM/TSP. In all the cases with CSF examinations, antiHTLV-1 antibodies and infected cells confirmed by PCR for tax were observed in the CSF.

Fourteen families presented clustering of IDH and/or HAM/TSP with diseases in two generations. However, in another family, clustering was observed in only one generation, involving a pair of siblings with IDH (Fig. 1).

Interestingly, among the 14 mothers with HAM/TSP, 12 had at least one child with HAM/TSP (Table 3 ). In contrast, only 3 of the 14 asymptomatic mothers had a child with $\operatorname{HAM} / \mathrm{TSP}(p=0.0018$, Fisher's exact test).

All the patients were from Bahia, Brazil, and had low socioeconomic status. The median age of the index cases at diagnosis of IDH ( $n=28$ ) was $2.1 \pm 1.1$ years (range: 3 months to 5 years). The median age of the index cases at diagnosis of HAM/TSP $(n=15)$ was $11.4 \pm 4$ years (range: $5-18$ ). The median age of the siblings at diagnosis of HAM/TSP $(n=9)$ was $16.8 \pm 7.7$ years (range: $7-30$ ). Fifty-five percent of the siblings with HAM/TSP were diagnosed in childhood and adolescence. The median age of the mothers at diagnosis of HAM/TSP was $41.5 \pm 8.2$ years (range: $28-56$ ). Among the IDH index cases and siblings with disease the male/female ratio was $1: 1.4$. Among siblings with only HAM/TSP the male/female ratio was 1:1.7.

\section{Discussion}

Fifty percent of the studied families presented clustering of IDH and/or HAM/TSP demonstrating a strong familial clustering of these diseases in Bahia, Brazil. We would like to emphasize that in our patients these diseases appeared mainly in childhood and adolescence, when the occurrence of HAM/TSP is rarely reported [1]. With the exception of one family described in Colombia [7], the other reports of the literature referred familial clustering only in

\section{Table 2}

Levels of ascertainment for diagnostic criteria of HTLV-1-associated myelopathy/tropical spastic paraparesis (HAM/TSP) [11].

\footnotetext{
Definite:

1. A non-remitting progressive spastic paraparesis with sufficiently impaired gait to be perceived by the patient. Sensory symptoms or signs may or may not be present. When present, they remain subtle and without a clear-cut sensory level. Urinary and anal sphincter signs or symptoms may or may not be present. 2. Presence of HTLV-I antibodies in serum and CSF confirmed by Western blot and/or a positive PCR for HTLV-I in blood and/or CSF.

3. Exclusion of other disorders that can resemble TSP/HAM.

Probable:

1. Monosymptomatic presentation: spacticity or hyperreflexia in the lower limbs or isolated Babinski sign with or without subtle sensory signs or symptoms, or neurogenic bladder only confirmed by urodynamic tests.

2. Presence of HTLV-I antibodies in serum and/or CSF confirmed by Western blot and/or a positive PCR for HTLV-I in blood and/or CSF

3. Exclusion of other disorders that can resemble TSP/HAM.

Possible:

1. Complete or incomplete clinical presentation.

2. Presence of HTLV-I antibodies in serum and/or CSF confirmed by Western blot and/or a positive PCR for HTLV-I in blood and/or CSF.

3. Disorders that can resemble TSP/HAM have not been excluded.
} 
$1 *$

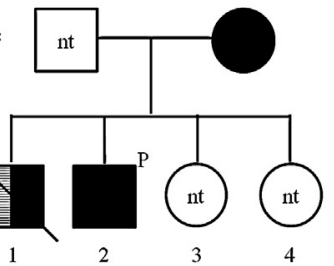

2

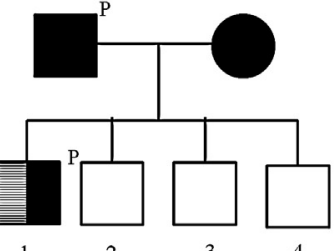

3

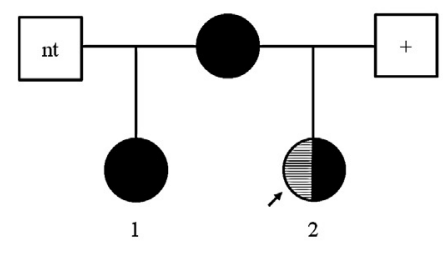

4

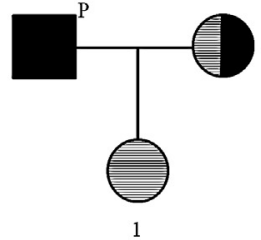

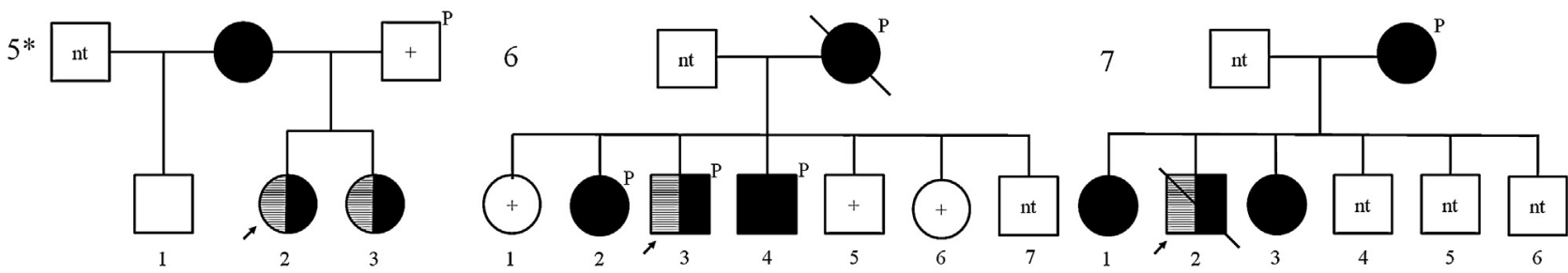
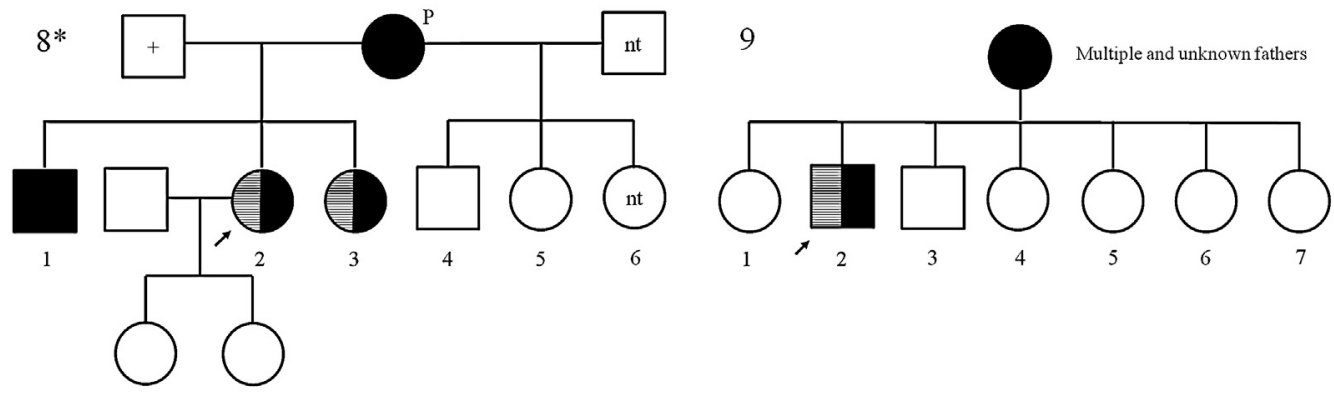

10
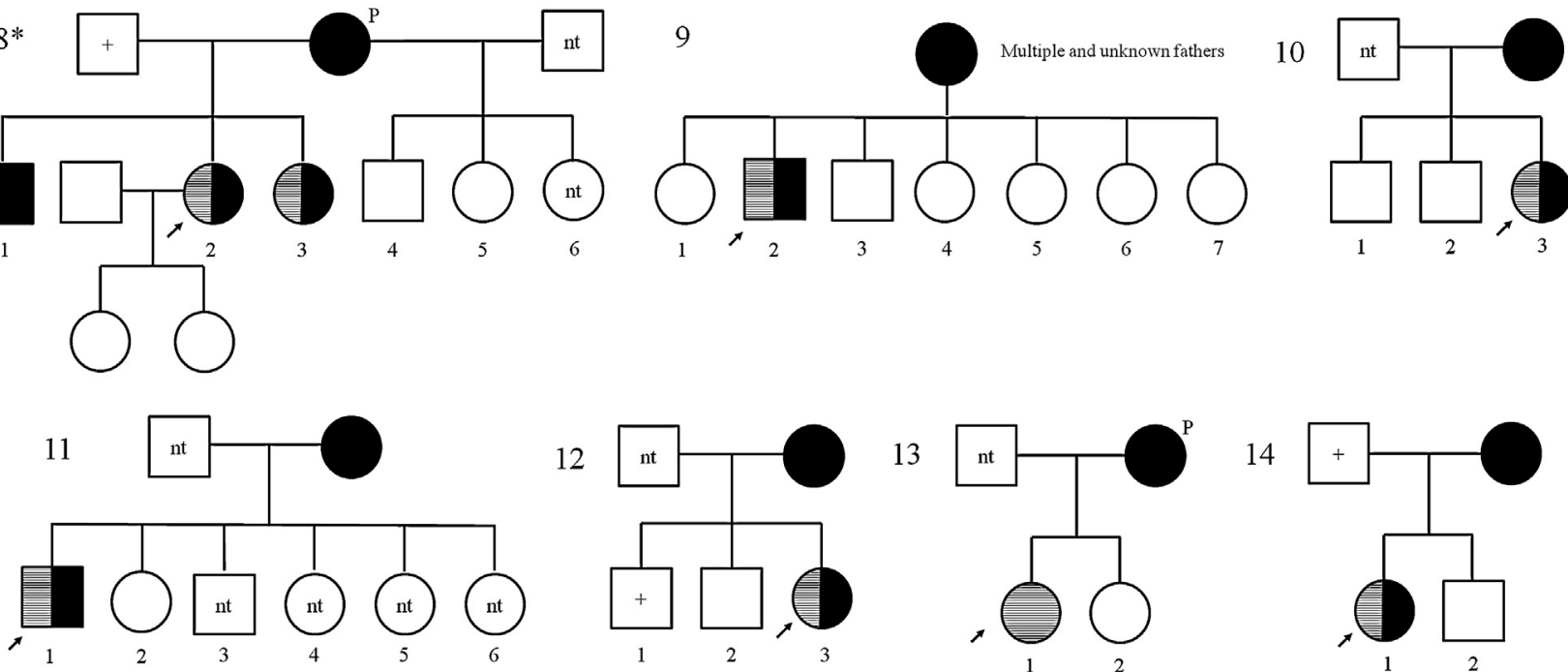

12

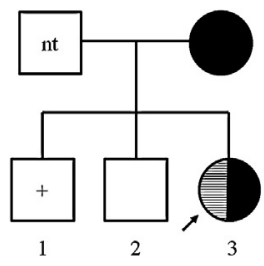

13

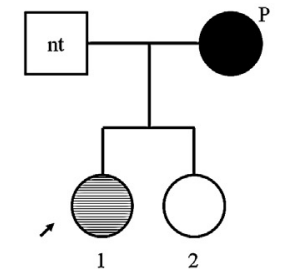

14

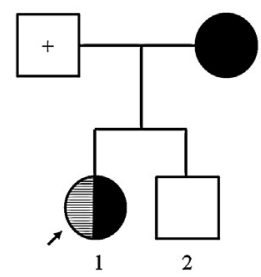

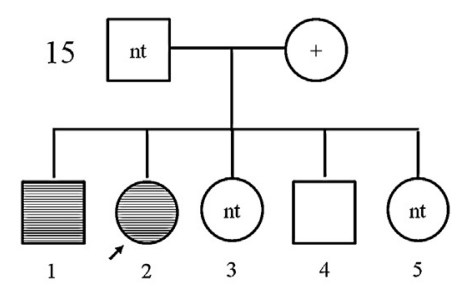

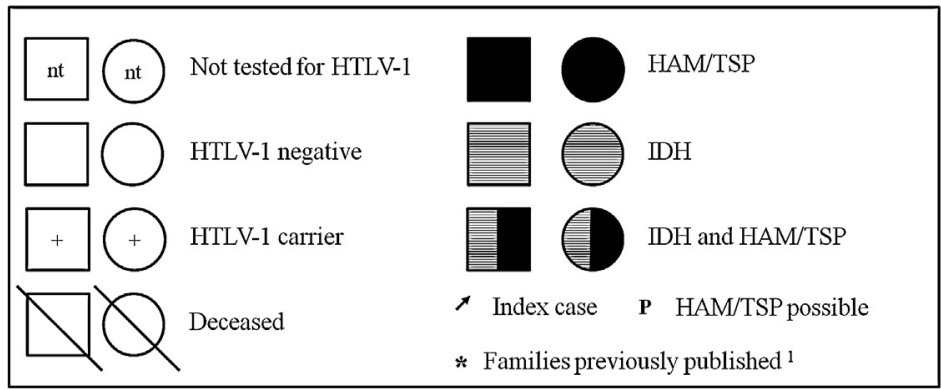

Fig. 1. Pedigree patterns of 15 families in which clustering of HAM/TSP and/or IDH was observed.

adulthood HAM/TSP. Clustering of IDH and HAM/TSP have only been referred in Jamaica, in one family, with the mother and one child with IDH, both presenting neurological manifestations, without diagnosis of definite HAM/TSP [12].
Except for two mothers, all the others with HAM/TSP had at least one child with HAM/TSP. This finding suggests that there may be a relationship between the presence of HAM/TSP in the mother and in her child. Considering that the proviral load is higher in HAM/TSP

Table 3

Proportion of mothers with or without HAM/TSP in relation with the occurrence of HAM/TSP in their children.

\begin{tabular}{|c|c|c|c|}
\hline Mothers & With children with HAM/TSP & With children without HAM/TSP & Total \\
\hline With HAM/TSP & $12(85.7 \%)$ & $2(14.3 \%)$ & 14 \\
\hline Without HAM/TSP & $3(21.4 \%)$ & $11(78.6 \%)$ & 14 \\
\hline Total & 15 & 13 & 28 \\
\hline
\end{tabular}


patients than in carriers [13], it is possible that a high quantity of HTLV-1 in the milk could increase the chance of development of HAM/TSP in these breast-fed children.

In order to better understand the clustering of IDH and HAM/TSP in those families, additional genetic studies will be performed in the members of these families with and without clustering, since it is known that some HLA alleles are related to the development of HAM/TSP $[12,14]$.

The limitation of this study was related to the poor participation of some of these families, all families had a low socioeconomic status and a low level of education. Because of this, we were not able to evaluate all the relatives and to perform the CSF examination of all patients with clinical evidence of HAM/TSP.

\section{Funding}

This study was supported by the Conselho Nacional de Pesquisa - CNPq. Prof. Achiléa Bittencourt is señior CNPq investigator.

\section{Competing interests}

All authors: no reported conflicts.

\section{Ethical approval}

The protocol of the present study was approved by the Research Ethics Committee of the Professor Edgard Santos Teaching Hospital. The parents or legal guardians of the children and adolescents gave their signed, informed consent for their participation. The work was conducted with integrity and in the line with accepted ethical principles. Also, steps were taken to protect patient privacy and confidentiality.

\section{Acknowledgment}

We thank Dr Vitoria Regina Almeida Rêgo, who provided us with facilities at the Dermatologic Clinic.

\section{References}

[1] Primo JR, Brites C, Oliveira MFSP, Moreno-Carvalho O, Machado M, Bittencourt AL. Infective dermatitis and juvenile HTLV-I associated myelopathy/tropical spastic paraparesis (HAM/TSP). Clinical Infectious Diseases 2005;41:535-41.

[2] Hanchard B. Outcomes of early life exposure to human T cell lymphotropic virus type 1. Clinical Infectious Diseases 2005;41:542-3.

[3] Oliveira MFSP, Fatal PL, Primo JR, Silva JLS, Batista ES, Farré L, et al. Infective dermatitis associated with human T-cell lymphotropic virus type 1: evaluation of 42 cases observed in Bahia, Brazil. Clinical Infectious Disease 2012;54:1714-9.

[4] Biglione MM, Pizarro M, Puca A, Salomón HE, Berría MI. A cluster of human T-cell lymphotropic virus type I-associated myelopathy/tropical spastic paraparesis in Jujuy, Argentina. Journal of Acquired Immune Deficiency Syndromes 2003;32:441-5.

[5] Kayembe K, Goubau P, Desmyter J, Vlietinck R, Carton H. A cluster of HTLV-1 associated tropical spastic paraparesis in Equateur (Zaire): ethnic and familial distribution. Journal of Neurology, Neurosurgery and Psychiatry 1990;53:4-10.

[6] Pombo-de-Oliveira MS, Carvalho SM, Borducchi D, Dobbin J, Salvador J, Correa RB, et al. Adult T-cell leukemia/lymphoma and cluster of HTLV-I associated diseases in Brazilian settings. Leukemia \& Lymphoma 2001;42:135-44.

[7] Mckhann G, Gibbs Jr CJ, Mora CA, Rodgers-Johnson PE, Liberski PP, Gdula WJ, et al. Isolation and characterization of HTLV-1 from symptomatic family members with tropical spastic paraparesis (HTLV-1 encephalomyeloneuropathy). Journal of Infectious Diseases 1989;160:371-9.

[8] Wilks RJ, La Grenade L, Hanchard B, Campbell M, Murphy, Cranston B, et al. Sibling adult T-cell leukemia/lymphoma and clustering of human T-cell lymphotropic virus Type I Infection in a Jamaican Family. Cancer 1993:72:2700-4.

[9] Plumelle Y, Pascaline N, Nguyen D, Panelatti G, Jouannelle A, Jouault H, et al. Adult T-cell leukemia-lymphoma: a clinico-pathologic study of twenty-six patients from Martinique. Hematologic Pathology 1993;7:251-62.

[10] Osame M. Review of WHO Kagoshima meeting and diagnostic guidelines for HAM/TSP. In: Blattner WA, editor. Human retrovirology: HTLV. New York: Raven Press; 1990. p. 191-7.

[11] Castro-Costa CM, Araújo AO Barreto MM, Takayanagui OM, Sohler MP da Silva EL, et al. Proposal for diagnostic criteria of tropical spastic paraparesis/HTLV1 -associated myelopathy (TSP/HAM). AIDS Research and Human Retroviruses 2006;22:931-5.

[12] La Grenade L, Sonoda S, Miller W, Pate E, Rodgers-Johnson P, Hanchard B, et al. HLA DRB1*DQB1* haplotype in HTLV-1-associated familial infective dermatitis may predict development of HTLV-1-associated myelopathy/tropical spastic paraparesis. American Journal of Medical Genetics 1996;61:31-7.

[13] Nagai M, Usuku K, Matsumoto W, Kodama D, Takenouchi N, Moritoyo T, et al. Analysis of HTLV-I proviral load in 202 HAM/TSP patients and 243 asymptomatic HTLV-I carriers: high proviral load strongly predisposes to HAM/TSP. Journal of Neurovirology 1998;4:586-93.

[14] Usuku K, Sonoda S, Osame M, Yashiki S, Takahashi K, Matsumoto M, et al. HLA haplotype-linked high imune responsiveness against HTLV-I in HTLVI-associated myelopathy: comparison with adult T-cell leukemia/lymphoma. Annals of Neurology 1988;23(Suppl. 1):S143-50. 